\title{
测墑补灌条件下高产小麦品种水分利用特性及干物质积累和分配
}

\author{
高春华 $^{1,2}$ 于振文 $^{1}$ 石 玉 $^{1, *}$ 张永丽 $^{1}$ 赵俊晔 $^{3}$
}

${ }^{1}$ 山东农业大学农业部作物生理生态与栽培重点开放实验室, 山东泰安 $271018 ;{ }^{2}$ 德州市农业科学研究院, 山东德州 $253051{ }^{3}$ 中国 农业科学院农业信息研究所, 北京 100081

摘 要: 2007-2009年连续2个小麦生长季, 利用测墑补灌技术, 设置 $0 ~ 140 \mathrm{~cm}$ 土壤相对含水量低(拔节期 $65 \%$, 开花 期 $55 \%$ 60\%)、中(拔节期 $75 \%$ ，开花期 65\% 70\%)、高(拔节期 75\%，开花期 $75 \%$ ) 3个处理，比较了 14 个小麦生产品种的 水分利用特性及干物质积累和分配的差异。以小麦籽粒产量和水分利用率为指标的聚类分析, 将 14 个小麦品种分为 3 组, 分别是超高产高水分利用率组(I 组)、超高产中水分利用率组(II 组)和高产低水分利用率组(III 组)。比较各组代表 品种的耗水量、耗水模系数及日耗水量, 播种至拔节期山农 15 (I 组)显著低于济麦22 (II 组)和烟农21 (III 组), 拔节至 开花期山农 15 显著高于济麦22和烟农 21 , 开花至成熟期品种间无显著差异。在中水分条件下, 山农 15 的土壤咜水消耗 量及其占总耗水量的比例显著高于济麦22和烟农 21 ，而在低和高水分条件下，3个品种无显著差异。在中、高水分条 件下，山农 15 开花期的干物质积累量显著高于济麦 22 和烟农 21 , 成熟期与济麦22无显著差异, 但显著高于烟农 $21 ;$ 营养器官开花前咜藏同化物向籽粒的转运量和转运率及对籽粒的贡献率均显著高于济麦22和烟农 $21 ; 3$ 个品种的经济 系数以山农 15 最大, 济麦22次之, 烟农 21 最小。

关键词：测墒补灌; 产量; 水分利用率; 干物质积累与分配

\section{Characteristics of Water Use and Dry Matter Accumulation and Distribution in Different High-yielding Wheat Cultivars under Supplemental Irrigation Based on Soil Moisture}

\author{
GAO Chun-Hua ${ }^{1,2}$, YU Zhen-Wen ${ }^{1}$, SHI Yu ${ }^{1, *}$, ZHANG Yong-Li ${ }^{1}$, and ZHAO Jun-Ye ${ }^{3}$ \\ ${ }^{1}$ Key Laboratory of Crop Ecophysiology and Cultivation, Ministry of Agriculture, Shandong Agricultural University, Tai'an 271018, China; \\ ${ }^{2}$ Academy of Agricultural Sciences of Dezhou City, Dezhou 253051, China; ${ }^{3}$ Agricultural Information Institute of Chinese Academy of Agricultural \\ Sciences, Beijing 100081, China.
}

\begin{abstract}
Water shortage is a serious problem threatening sustainable agricultural development in the North China Plain, where winter wheat (Triticum aestivum L.) is the largest water-consuming crop. The objective of this study was to guide wheat production in this area by selecting high water efficient cultivar and improving irrigation regime. In a two-year field experiment from autumn of 2007 to summer of 2009, irrigation quantum was controlled based on testing soil moisture (SM) in 0-140 cm depth, which was designed in low ( $\mathrm{SM}$ of $65 \%$ at jointing and $55 \%-60 \%$ at anthesis stage), medium (SM of $75 \%$ at jointing and $65 \%-70 \%$ at anthesis stage), and high (SM of $75 \%$ at jointing and $75 \%$ at anthesis stage) levels. Water use efficiency (WUE), dry matter accumulation and distribution in wheat plant, and grain yield were tested and compared among 14 commercial cultivars. Based on grain yield and WUE, the 14 cultivars were clustered into three groups, namely, super-high yield and high WUE group (I), super-high yield and medium WUE group (II), and high yield and low WUE group (III). One representative cultivar was selected from each group to compare the amount and proportion of water consumption during sowing-jointing, jointing-anthesis, and anthesis-maturity periods. Shannong 15 from group I had significantly lower water consumption from sowing to jointing than Jimai 22 from group II and Yannong 21 from group III, and significantly higher water consumption from jointing to anthesis. However, water consumption amount and proportion had no significant differences among the three cultivars from anthesis to
\end{abstract}

本研究由国家自然科学基金项目(31171498), 山东省自然科学基金项目(ZR2011CQ014), 高等学校博士学科点专项科研基金(新教师类) (20123702120014)和山东农业大学博士基金项目资助。

“通讯作者(Corresponding author): 石玉, E-mail: shiyu@sdau.edu.cn, Tel: 0538-8241484

第一作者联系方式: E-mail: chunhuaaa009@163.com

Received(收稿日期): 2013-03-26; Accepted(接受日期): 2013-07-25; Published online(网络出版日期): 2013-09-29.

URL: http://www.cnki.net/kcms/detail/11.1809.S.20130929.1536.006.html 
maturity. Under medium SM condition, soil water consumption in Shannong 15 was significantly higher than that in Jimai 22 and Yannong 21, but such advantage in Shannong 15 disappeared under high SM condition. Under medium and high SM conditions, translocation amount and ratio of dry matter accumulated before anthesis and its contribution to grain were significantly higher in Shannong 15 than in Jimai 22 and Yannong 21. Among the three cultivars, harvest index was the highest in Shannong 15, the medium in Jimai 22, and the lowest in Yannong 21.

Keywords: Irrigation based on testing soil moisture; Yield; Water use efficiency; Dry matter accumulation and distribution

我国人均水资源拥有量为世界平均值的 $1 / 4$, 是 世界上13个严重缺水的国家之一 ${ }^{[1]}$ 。华北小麦主产 区水资源的严重匮乏已经成为该地区小麦生产的主 要限制因素, 如何合理利用有限的水资源、减少灌 溉用水、提高水分利用率是小麦生产迫切需要解决

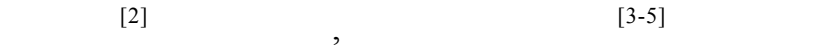
期 ${ }^{[6-7]}$ 、灌水方法 ${ }^{[8-9]}$ 形成适度水分胁迫, 小麦可获得 较高的产量和水分利用率, 达到节水高产的目的。

水高效生理和遗传机制在不同小麦品种间差异 较大 ${ }^{[10-11]}$, 小麦籽粒产量差异可达 $44.9 \%$, 水分利用 率差异为 $42.2 \%{ }^{[12]}$ 。多穗型小麦品种叶片对籽粒产量 的贡献大于大穗型品种 ${ }^{[13]}$, 大穗型品种在灌浆中后 期比多穗型品种具有更强的淀粉合成能力, 但对水 分较为敏感 ${ }^{[14]}$ 。旱地品种一般在不灌溉条件下水分 利用率较高; 水旱兼用型品种在灌溉较少(返青后灌 水 1 次)条件下水分利用率最大; 而水地品种则一般 在充分灌溉(返青后灌水2 3次)条件下，水分利用率 和产量协同达到最优 ${ }^{[15]}$ 。

节水灌溉是华北平原小麦高产栽培中推广的关 键技术之一, 但是高产条件下不同水浇地品种在各 生育阶段的耗水特性、水分利用率及干物质积累与 分配的差异尚不清楚。本试验以生产中推广的14个 小麦品种为材料, 采用测墑补灌的方法, 研究不同 高产品种的水分利用特性及干物质积累与分配的差 异, 为小麦生产中选择高产和水分利用率高的品 种、完善高产节水栽培技术体系提供理论依据。

\section{1 材料与方法}

\section{1 试验地概况}

山东泰安山东农业大学试验农场地处暖温带大 陆性半湿润季风气候, 年均气温 $13^{\circ} \mathrm{C}$, 年均降水量 $675.3 \mathrm{~mm}$ 。试验地为沙壤土, 小麦播种前 $0 \sim 20 \mathrm{~cm}$ 土 层含有机质 $1.36 \%$ 、全氮 $0.85 \%$ 、碱解氮 $79.40 \mathrm{mg}$ $\mathrm{kg}^{-1}$ 、速效磷 $38.20 \mathrm{mg} \mathrm{kg}^{-1}$ 、速效钾 $80.00 \mathrm{mg} \mathrm{kg}^{-1}$ 。 $0 \sim 20 、 20 \sim 40 、 40 \sim 60 、 60 \sim 80 、 80 \sim 100 、 100 \sim 120$ 、 120 140、140 160、160 180和180 200 cm土层土壤 田间持水量分别为 $25.92 \% 、 25.11 \%$ 、 $24.35 \%$ 、 $25.20 \% 、 24.56 \% 、 24.05 \% 、 24.98 \% 、 24.87 \% 、 25.01 \%$
和 $24.76 \%$, 土壤容重分别为 $1.51 、 1.52 、 1.54 、 1.56$ 、 $1.55 、 1.57 、 1.57 、 1.58 、 1.59$ 和 $1.59 \mathrm{~g} \mathrm{~cm}^{-3}$ 。

\section{2 试验材料}

2007 - 2008 年度小麦生长季, 选用 14 个山东省 选育的生产品种，分别是良星 99、烟农 21、烟 2415、 烟 5158、烟 5286、山农 15、洲元 9369、济麦 22、 泰 9818、临麦 4 号、汶农 6 号、潍麦 8 号、聊麦 19 和山农 8355。以籽粒产量和水分利用率为指标, 对 这 14 个品种作聚类分析, 将其分为 3 组。20082009 年度每组选择 1 个代表性品种(山农 15、济麦 22 和烟农 21)作进一步试验。

\section{3 试验设计}

按 $0 \sim 140 \mathrm{~cm}$ 土壤平均相对含水量设置低土壤含 水量(LSM)、中土壤含水量(MSM)和高土壤含水量 (HSM) 3 个水分处理, 两年度自然降水和灌溉量见 表 1 。用土钻取0 $200 \mathrm{~cm}$ 土层, 每 $20 \mathrm{~cm}$ 为一层, 将样 品立即装入铝盒, 称鲜质量; 然后 $110^{\circ} \mathrm{C}$ 烘干至恒质 量, 称干质量。土壤质量含水量 $(\%)=$ (土壤鲜质量 土壤干质量 $) /$ 土壤干质量 $\times 100$; 土壤相对含水量 $(\%)=$ 土壤质量含水量/田间持水量 $\times 100$; 用环刀法 ${ }^{[16]}$ 测定田间持水量。灌水量 $(\mathrm{mm})$ 由 $m=10 \times \rho b \times H \times\left(\beta_{i}\right.$ $\left.-\beta_{j}\right)$ 计算得出 ${ }^{[17]}$, 式中 $m$ 为灌水量 $(\mathrm{mm}), H$ 为该时段 土壤计划湿润层的深度(本试验为 $140 \mathrm{~cm}$ ), $\rho b$ 为计划 湿润层内土壤容重 $\left(\mathrm{g} \mathrm{cm}^{-3}\right), \beta_{i}$ 为设计含水量(田间持 水量乘以目标相对含水量), $\beta_{j}$ 为自然含水量, 即灌 溉前土壤含水量。用水表计灌水量。

采用裂区设计, 主区为土壤相对含水量, 副区 为品种，小区间设 $1.0 \mathrm{~m}$ 隔离区，小区面积均为 $4 \mathrm{~m} \times$ $4 \mathrm{~m}=16 \mathrm{~m}^{2}, 3$ 次重复。2007年10月10日播种, 2008年6 月 10 日收获; 2008 年 10 月 8 日播种, 2009年6月 10 日收 获。四叶期定苗至195株 $\mathrm{m}^{-2}$ 。两年度均在播种前施 纯氮 $105 \mathrm{~kg} \mathrm{hm}^{-2} 、 \mathrm{P}_{2} \mathrm{O}_{5} 112.5 \mathrm{~kg} \mathrm{hm}^{-2}$ 和 $\mathrm{K}_{2} \mathrm{O} 112.5 \mathrm{~kg}$ $\mathrm{hm}^{-2}$, 拔节期开沟追施纯氮 $135 \mathrm{~kg} \mathrm{hm}^{-2}$ 。生长期按当 地高产田进行田间管理。

\section{4 耗水量计算方法}

测定0 200 $\mathrm{cm}$ 土层土壤水分含量变化，根据 SPAC理论用农田水分平衡法 ${ }^{[18]}$ 计算耗水量。 
表 $12007-2008$ 和 2008-2009 两年度自然降水量和灌溉量

Table 1 Precipitations and irrigation quantities in 2007-2008 and 2008-2009 wheat growing seasons (mm)

\begin{tabular}{|c|c|c|c|}
\hline \multicolumn{2}{|c|}{ 生育阶段 Growing period } & $2007-2008$ & 2008-2009 \\
\hline \multicolumn{4}{|c|}{ 降水量 Precipitation } \\
\hline \multicolumn{2}{|c|}{ 播种-越冬前 Sowing-before overwintering } & 28.3 & 14.8 \\
\hline \multicolumn{2}{|c|}{ 越冬前-返青 Before overwintering-regreening } & 15.1 & 10.9 \\
\hline \multicolumn{2}{|c|}{ 返青-拔节 Regreening-jointing } & 17.7 & 18.0 \\
\hline \multicolumn{2}{|c|}{ 拔节-开花 Jointing-anthesis } & 68.2 & 54.0 \\
\hline \multicolumn{2}{|c|}{ 开花-成熟 Anthesis-maturity } & 40.5 & 47.0 \\
\hline \multicolumn{4}{|c|}{ 灌水量 Irrigation } \\
\hline \multirow[t]{2}{*}{ LSM } & 拔节期 Jointing & 0 & 0 \\
\hline & 开花期 Anthesis & 0 & 0 \\
\hline \multirow[t]{2}{*}{ MSM } & 拔节期 Jointing & $53.8-65.8^{\text {a) }}$ & $66.6-70.3^{\mathrm{c})}$ \\
\hline & 开花期 Anthesis & 0 & 0 \\
\hline \multirow[t]{2}{*}{ HSM } & 拔节期 Jointing & $53.8-65.8^{\text {a) }}$ & $66.6-70.3^{\mathrm{c})}$ \\
\hline & 开花期 Anthesis & $53.3-63.6^{\mathrm{b})}$ & $27.1-40.0^{\mathrm{d})}$ \\
\hline
\end{tabular}

低(LSM)、中(MSM)、高土壤含水量(HSM)处理均为 $0 \sim 140 \mathrm{~cm}$ 土壤平均相对含水量, 2007-2008 年度依次为拔节期 $65 \%+$ 开花期 $55 \%$ 、 拔节期 75\%+开花期 65\%和拔节期 75\%+开花期 75\%, 2008-2009 年度依次为拔节期 65\%+开花期 60\%、拔节期 75\%+开花期 70\%和拔 节期 75\%+开花期 75\%。2007-2008 年度, MSM 处理的开花期自然含水量已达到设计要求 $(65 \%)$, 所以开花期未补灌。2008-2009 年度, MSM 处理开花期土壤含水量高于上年度设计水平，是由于该年度开花期前降雨导致开花期土壤相对含水量较高，不补灌也达到 $70 \%$ 。 2007-2008 年度，14 个品种(良星 99、烟农 21、烟 2415、烟 5158、烟 5286、山农 15、洲元 9369、济麦 22、泰 9818、临麦 4 号、汶 农 6 号、潍麦 8 号、聊麦 19 和山农 8355)的实际灌水量分别为 ${ }^{a)} 65.8 、 61.1 、 58.4 、 59.9 、 53.8 、 57.3 、 65.3 、 61.1 、 59.9 、 65.3 、 53.8 、$ $60.2 、 61.1$ 和 $58.4 \mathrm{~mm} ;{ }^{\text {b) }} 56.8 、 56.8 、 55.6 、 55.6 、 57.7 、 57.5 、 58.9 、 63.6 、 53.3 、 57.7 、 57.5 、 56.8 、 56.8$ 和 $57.6 \mathrm{~mm}$ 。2008-2009 年 度, 3 个品种(山农 15、济麦 22 和烟农 21)的实际灌水量分别为 ${ }^{\mathrm{c})} 66.6 、 70.3$ 和 $67.0 \mathrm{~mm} ;{ }^{\mathrm{d})} 40.0 、 31.7$ 和 $27.1 \mathrm{~mm}$ 。

Low (LSM), medium (MSM), and high soil moisture (HSM) treatments were designed according to the relative soil water content in $0-140$ soil layers, with soil moisture (SM) at joining + anthesis of $65 \%+55 \%, 75 \%+65 \%$, and $75 \%+75 \%$ in $2007-2008$ growing season and $65 \%+60 \%, 75 \%+70 \%$, and $75 \%+75 \%$ in $2008-2009$ growing season, respectively. In the 2007-2008 growing season, zero irrigation was conducted at anthesis stage in MSM treatment because natural SM had reached the designed level (65\%). The designed SM at anthesis in 2008-2009 was higher than that in 2007-2008, because precipitation occurred before anthesis, which resulted in SM of 70\% without supplementary irrigation. The actual amounts of irrigation for 14 cultivars (Liangxing 99, Yannong 21, Yan 2415, Yan 5158, Yan 5286, Shannong 15, Zhouyuan 9369, Jimai 22, Tai 9818, Linmai 4, Wennong 6, Weimai 8, Liaomai 19, and Shannong 8355) used in 2007-2008 were ${ }^{\text {a) }} 65.8$, $61.1,58.4,59.9,53.8,57.3,65.3,61.1,59.9,65.3,53.8,60.2,61.1$, and $58.4 \mathrm{~mm}$, respectively; ${ }^{\text {b) }} 56.8,56.8,55.6,55.6,57.7,57.5,58.9,63.6$, $53.3,57.7,57.5,56.8,56.8$, and $57.6 \mathrm{~mm}$, respectively. The actual amounts of irrigation for 3 cultivars (Shannong 15, Jimai 22, and Yannong 21) used in 2008-2009 were ${ }^{\mathrm{c})} 66.6,70.3$, and $67.0 \mathrm{~mm}$, respectively; ${ }^{\text {d) }} 40.0,31.7$, and $27.1 \mathrm{~mm}$, respectively.

作物生育期耗水量:

$E T \alpha=P+U-R-F+\Delta W+I$

式中, $\Delta W$ 为土壤咜水消耗量; $P$ 为该时段降水量 $(\mathrm{mm}) ; U$ 为地下水通过毛管作用上移补给作物水量 $(\mathrm{mm}) ; R$ 为地表径流量 $(\mathrm{mm}) ; F$ 为补给地下水量 $(\mathrm{mm})$; $I$ 为灌水量 $(\mathrm{mm})$ 。本试验地块地势平坦, 地下水埋深 $5 \mathrm{~m}$ 以下, 降水入渗深度不超过 $2 \mathrm{~m}$, 因此 $U 、 R 、 F$ 均 为 0 ; 本试验以 $20 \mathrm{~cm}$ 为一个土壤层次。

$$
E T_{1-2}=10 \sum_{i=1}^{n} \gamma_{i} H_{i}\left(\theta_{i 1}-\theta_{i 2}\right)+I+P
$$

式中, $i$ 为土层编号; $n$ 为总土层数; $\gamma_{i}$ 为第 $i$ 层土壤容 重; $H_{i}$ 为第 $i$ 层土壤厚度; $\theta_{i 1}$ 和 $\theta_{i 2}$ 为第 $i$ 层土壤时段初 和时段末的含水量, 以占干土重的百分数计; I为时 段内的灌水量; $P$ 为该时段内的降水量。

\section{5 干物质转运和水分利用率计算方法} 开花期按叶、茎+叶鞘和穗取样，成熟期按叶、
茎+叶鞘、穗轴+颖壳和籽粒取样, 3 次重复, $75^{\circ} \mathrm{C}$ 烘至 恒重, 称干重, 计算同化物转运量和转运率 ${ }^{[19]}$ 。营养 器官开花前咜藏同化物转运量 $=$ 开花期干重 - 成熟期 干重; 营养器官开花前䛎藏同化物转运率 $(\%)=$ (开 花期干重-成熟期干重)/开花期干重 $\times 100$; 开花后同 化物输入籽粒量=成熟期籽粒干重-营养器官花前䞎 藏物质转运量; 对籽粒产量的贡献率 $(\%)=$ 开花前营 养器官咜藏物质转运量/成熟期籽粒干重 $\times 100$ 。水分 利用率 $\left(\mathrm{kg} \mathrm{hm}^{-2} \mathrm{~mm}^{-1}\right)=$ 籽粒产量 $\left(\mathrm{kg} \mathrm{hm}^{-2}\right) /$ 作物全 生育期耗水量 $(\mathrm{mm})^{[20]}$ 。

\section{6 数据分析}

用Microsoft Excel 2003处理数据和绘图，用 DPS7.5软件进行数据统计分析和差异显著性检验, 用DPS7.5数据分析软件进行数据标准化转化, 根据 欧氏距离的大小, 运用最长距离法, 对供试品种作 聚类分析。 


\section{2 结果与分析}

2.1 不同小麦品种籽粒产量及水分利用率的差异 以2007-2008年度生长季籽粒产量和水分利用 率为指标, 通过聚类分析将 14 个品种分成3组(图 1), 分别是超高产高水分利用率组(I)、超高产中水分利 用率组(II)和高产低水分利用率组(III)。本文将单产 超过9000 kg hm${ }^{-2}$ 的品种称为超高产品种 ${ }^{[21]}$, 单产 $7500 \sim 9000 \mathrm{~kg} \mathrm{hm}^{-2}$ 的称为高产品种。

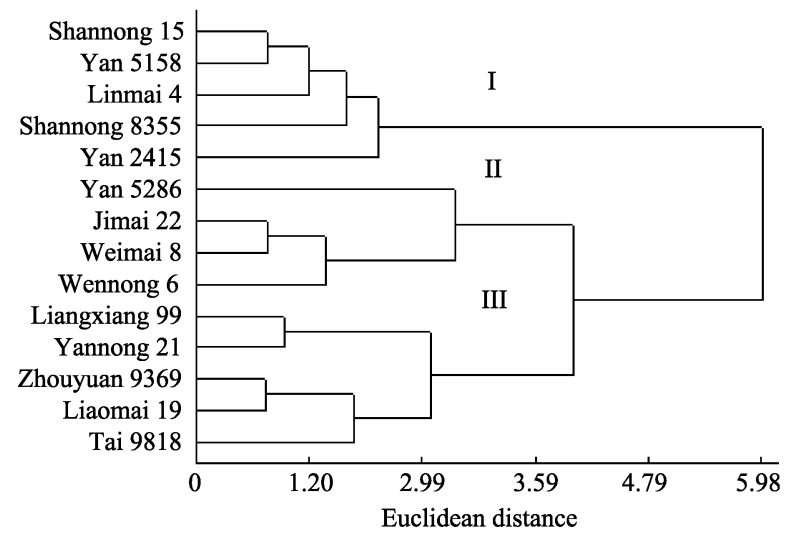

图 1 基于籽粒产量和水分利用率的品种聚类图

Fig. 1 Dendrogram of wheat cultivars based on grain yield and water use efficiency

I：超高产高水分利用率组; II：超高产中水分利用率组; III：高 产低水分利用率组。

I: super-high yield and high WUE group; II: super-high yield and medium WUE group; III: high yield and low WUE group.

随开花期土壤相对含水量增加，水分利用率呈 降低的趋势。I和III组品种的籽粒产量平均值在MSM 和HSM处理间无显著差异, 均显著高于LSM处理; 而II组各品种籽粒产量则表现为HSM > MSM > LSM，处理间有显著差异(表2)。表明不同组别获得 超高产或高产的需水特性存在差异, I组在开花期土 壤含水量较低条件下即能获得超高产, 而II组开花 期要求的土壤相对含水量较高。

2008-2009年度, 对3个代表性品种的籽粒产 量和水分利用率进行了测定, 其结果趋势与上年度 相同(表3), 因此以这3个品种分别代表其所在组进 行水分利用特性及干物质积累分配的分析。

2.2 不同小麦品种耗水量的水分来源及其占总 耗水量的比例

随开花期土壤相对含水量增加, 3 个品种均表现 为总耗水量增加, 土壤咜水消耗量及其占总耗水量 的比例降低, 灌溉水占总耗水量的比例增加(表4), 表明降低开花期土壤相对含水量有利于小麦利用降
水和土壤咜水。

在 LSM 和 HSM 条件下, 3 个品种的总耗水量及 土壤水消耗量占总耗水量的比例均无显著差异; 在 MSM 条件下, 山农 15 的总耗水量、土壤水消耗量 及占总耗水量的比例均显著高于济麦 22 和烟农 21 , 表明在 MSM 条件下, 山农 15 有较高的利用土壤䛎 水能力。

2.3 不同小麦品种各生育阶段的耗水量、耗水模 系数和日耗水量

不同处理播种至拔节期的耗水量和日耗水量无 显著差异, 耗水模系数为 $\mathrm{LSM}>\mathrm{MSM}>\mathrm{HSM}$; 拔节至 开花期, 耗水量、耗水模系数和日耗水量在MSM和 HSM处理间无显著差异，显著高于LSM处理; 开花 至成熟期的耗水量、耗水模系数和日耗水量均为 HSM处理最高(表5)。不同土壤相对含水量对 3 个品 种的拔节至开花期和开花至成熟期耗水特性的调控 效应不同, 可能是导致不同品种各处理的总耗水量 不同的主要原因。

在同一处理中，山农 15 播种至拔节期的耗水 量、耗水模系数及日耗水量显著低于济麦 22 和烟农 21 ，拔节至开花期显著高于济麦22和烟农 21 ，开花 至成熟期与济麦22和烟农 21 无显著差异(表5)。表明 不同品种在各个生育阶段的耗水强度存在差异, 山 农15拔节前耗水量少，拔节至开花期通过高效吸收 土壤㲸水提高了耗水量, 有利于此阶段小花的成熟 发育，防止小花退化，提高山农 15 的分藍成穗率和 穗粒数。

\section{4 不同小麦品种干物质积累及分配}

3 个品种开花期和成熟期干物质积累量均为 MSM 和 HSM 处理无显著差异, 显著高于 LSM 处 理。山农 15 和烟农 21 的经济系数为 $\mathrm{LSM}>\mathrm{MSM}>$ HSM，处理间差异显著; 济麦22为 LSM 显著高于 MSM 和 HSM 处理, MSM 和 HSM 处理间无显著差 异(表6)。表明灌溉降低了成熟期干物质积累量向籽 粒的分配比例。随开花期土壤相对含水量提高, 营 养器官开花前䇄藏同化物向籽粒的转运量和转运率 及对籽粒的贡献率均显著降低，表现为 LSM $>$ MSM $>$ HSM, 开花后干物质积累量及其对籽粒的贡 献率则相反。表明提高开花期土壤相对含水量促进 了开花后干物质积累及向籽粒转运, 不利于开花前 悾藏同化物向籽粒的转运。

在 LSM 条件下，山农15和济麦22开花期和成熟 期干物质积累量均显著高于烟农21；MSM 和 HSM 
表 2 不同小麦品种籽粒产量和水分利用率(2007-2008)

Table 2 Grain yield and water use efficiency of different wheat cultivars in 2007-2008

\begin{tabular}{|c|c|c|c|c|c|c|c|}
\hline \multirow{2}{*}{$\begin{array}{c}\text { 组 } \\
\text { Group }\end{array}$} & \multirow{2}{*}{$\begin{array}{c}\text { 品种 } \\
\text { Cultivar }\end{array}$} & \multicolumn{2}{|c|}{ LSM } & \multicolumn{2}{|c|}{ MSM } & \multicolumn{2}{|c|}{ HSM } \\
\hline & & $\begin{array}{c}\mathrm{GY} \\
\left(\mathrm{kg} \mathrm{hm}^{-2}\right)\end{array}$ & $\begin{array}{c}\text { WUE } \\
\left(\mathrm{kg} \mathrm{hm}^{-2} \mathrm{~mm}^{-1}\right)\end{array}$ & $\begin{array}{c}\mathrm{GY} \\
\left(\mathrm{kg} \mathrm{hm}^{-2}\right)\end{array}$ & $\begin{array}{c}\text { WUE } \\
\left(\mathrm{kg} \mathrm{hm}^{-2} \mathrm{~mm}^{-1}\right)\end{array}$ & $\begin{array}{c}\mathrm{GY} \\
\left(\mathrm{kg} \mathrm{hm}^{-2}\right)\end{array}$ & $\begin{array}{c}\text { WUE } \\
\left(\mathrm{kg} \mathrm{hm}^{-2} \mathrm{~mm}^{-1}\right)\end{array}$ \\
\hline \multirow[t]{5}{*}{ I } & 山农 15 Shannong 15 & 8395.0 & 22.4 & 9074.8 & 21.9 & 9221.4 & 20.6 \\
\hline & 烟 5158 Yan 5158 & 8361.9 & 22.1 & 9014.5 & 21.8 & 9292.6 & 21.9 \\
\hline & 临麦 4 号 Linmai 4 & 8544.4 & 22.2 & 9109.0 & 22.1 & 9602.2 & 20.2 \\
\hline & 山农 8355 Shannong 8355 & 8274.4 & 22.5 & 9330.8 & 21.5 & 9092.7 & 20.4 \\
\hline & 烟 2415 Yan 2415 & 8574.6 & 22.9 & 9116.6 & 21.8 & 9185.9 & 20.9 \\
\hline \multirow[t]{4}{*}{ II } & 烟 5286 Yan 5286 & 7633.7 & 19.9 & 8341.2 & 20.5 & 9313.0 & 20.4 \\
\hline & 济麦 22 Jimai 22 & 8061.1 & 21.6 & 8465.2 & 20.0 & 9171.7 & 20.1 \\
\hline & 潍麦 8 号 Weimai 8 & 8096.2 & 21.3 & 8679.3 & 20.4 & 9057.5 & 20.1 \\
\hline & 汶农 6 号 Wennong 6 & 8427.8 & 21.5 & 8523.0 & 20.9 & 9097.3 & 19.8 \\
\hline \multirow[t]{5}{*}{ III } & 良星 99 Liangxing 99 & 8108.8 & 20.3 & 8525.6 & 20.7 & 8455.3 & 18.6 \\
\hline & 烟农 21 Yannong 21 & 7887.8 & 20.5 & 8521.6 & 20.0 & 8413.3 & 18.5 \\
\hline & 洲元 9369 Zhouyuan 9369 & 7697.3 & 20.3 & 8131.1 & 19.0 & 8355.6 & 18.6 \\
\hline & 聊麦 19 Liaomai 19 & 7638.6 & 20.1 & 8198.1 & 18.9 & 8156.3 & 19.1 \\
\hline & 泰 9818 Tai 9818 & 7363.6 & 19.8 & 7974.0 & 20.1 & 8429.9 & 18.7 \\
\hline \multicolumn{2}{|c|}{ I 组平均 I group average } & $8430.1 \mathrm{a}$ & $22.4 \mathrm{a}$ & $9129.1 \mathrm{a}$ & $21.8 \mathrm{a}$ & $9279.0 \mathrm{a}$ & $20.8 \mathrm{a}$ \\
\hline \multicolumn{2}{|c|}{ II 组平均 II group average } & $8054.7 \mathrm{~b}$ & $21.1 \mathrm{~b}$ & $8502.2 \mathrm{~b}$ & $20.5 \mathrm{~b}$ & 9159.9 a & $20.1 \mathrm{~b}$ \\
\hline \multicolumn{2}{|c|}{ III 组平均 III group average } & $7739.2 \mathrm{c}$ & $20.2 \mathrm{c}$ & $8270.1 \mathrm{c}$ & $19.7 \mathrm{c}$ & $8362.1 \mathrm{~b}$ & $18.7 \mathrm{c}$ \\
\hline
\end{tabular}

LSM：低土壤含水量; MSM: 中土壤含水量; HSM: 高土壤含水量; GY：籽粒产量; WUE：水分利用效率。平均值后不同字母表示 相同土壤水分条件下品种组间差异达 0.05 显著水平。

LSM: low soil moisture; MSM: medium soil moisture; HSM: high soil moisture; GY: grain yield; WUE: water use efficiency. Different letters after averages indicate significant difference $(P<0.05)$ among cultivar groups under the same soil moisture.

表 3 不同小麦品种籽粒产量和水分利用率(2008-2009)

Table 3 Grain yield and water use efficiency of different wheat cultivars in 2008-2009

\begin{tabular}{|c|c|c|c|c|c|c|}
\hline \multirow{2}{*}{$\begin{array}{c}\text { 品种 } \\
\text { Cultivar }\end{array}$} & \multicolumn{2}{|r|}{ LSM } & \multicolumn{2}{|r|}{ MSM } & \multicolumn{2}{|r|}{ HSM } \\
\hline & GY $\left(\mathrm{kg} \mathrm{hm}^{-2}\right)$ & WUE $\left(\mathrm{kg} \mathrm{hm}^{-2} \mathrm{~mm}^{-1}\right)$ & $\mathrm{GY}\left(\mathrm{kg} \mathrm{hm}^{-2}\right)$ & WUE $\left(\mathrm{kg} \mathrm{hm}^{-2} \mathrm{~mm}^{-1}\right)$ & GY $\left(\mathrm{kg} \mathrm{hm}^{-2}\right)$ & WUE $\left(\mathrm{kg} \mathrm{hm}^{-2} \mathrm{~mm}^{-1}\right)$ \\
\hline $\begin{array}{c}\text { 山农 } 15 \\
\text { Shannong } 15\end{array}$ & 8082.9 a & $21.7 \mathrm{a}$ & $9163.4 \mathrm{a}$ & 20.9 a & $9182.1 \mathrm{a}$ & $20.4 \mathrm{a}$ \\
\hline $\begin{array}{l}\text { 济麦 } 22 \\
\text { Jimai } 22\end{array}$ & $7538.7 \mathrm{~b}$ & $20.4 \mathrm{~b}$ & $8660.3 \mathrm{~b}$ & $20.1 \mathrm{~b}$ & $9005.8 \mathrm{a}$ & $19.8 \mathrm{~b}$ \\
\hline $\begin{array}{c}\text { 烟农 } 21 \\
\text { Yannong } 21\end{array}$ & $7326.6 \mathrm{c}$ & $19.7 \mathrm{c}$ & $8150.6 \mathrm{c}$ & $19.3 \mathrm{c}$ & $8317.7 \mathrm{~b}$ & $18.8 \mathrm{c}$ \\
\hline
\end{tabular}

LSM：低土壤含水量; MSM：中土壤含水量; HSM：高土壤含水量; GY：籽粒产量; WUE：水分利用效率。数据后不同字母表示相 同土壤水分条件下品种间差异达 0.05 显著水平。

LSM: low soil moisture; MSM: medium soil moisture; HSM: high soil moisture; GY: grain yield; WUE: water use efficiency. Different letters after data indicate significant difference $(P<0.05)$ among cultivars under the same soil moisture.

条件下，山农 15 开花期干物质积累量显著高于济麦 22 和烟农 21 ，成熟期则表现为山农 15 和济麦 22 显著 高于烟农 21 。LSM条件下, 经济系数为山农 $15>$ 烟农 $21>$ 济麦 22, MSM和HSM条件下, 经济系数为山农 $15>$ 济麦22>烟农 21 , 各品种差异显著(表6), 表明产 量和水分利用率高的品种其经济系数亦较高。各水 分处理下，山农 15 营养器官开花前冮藏同化物向籽
粒的转运量和转运率及对䊏粒的贡献率均显著高于 济麦22和烟农 21 , 开花后干物质积累量及其对䊏粒 的贡献率则相反。表明山农 15 开花期和成熟期均具 有较高的干物质积累量, 经济系数较高, 且开花期 前䇄藏同化物向籽粒的转运能力较强, 为其获得超 高产奠定了物质基础。

综合分析耗水特性可见，拔节至开花期耗水量 
表 4 不同小麦品种的耗水来源及其占总耗水量的比例(2008-2009)

Table 4 Source of water consumed during wheat growth and its proportion to total water consumption in different cultivars in 2008-2009

\begin{tabular}{|c|c|c|c|c|c|c|c|}
\hline \multirow{2}{*}{$\begin{array}{c}\text { 处理 } \\
\text { Treatment }\end{array}$} & \multirow{2}{*}{$\begin{array}{c}\text { 总耗水量 } \\
\text { Total water } \\
\text { consumption }(\mathrm{mm})\end{array}$} & \multicolumn{2}{|c|}{ 土壤水消耗 Soil water consumption } & \multicolumn{2}{|c|}{ 灌溉 Irrigation } & \multicolumn{2}{|c|}{ 降水 Precipitation } \\
\hline & & $\begin{array}{c}\text { 消耗量 } \\
\text { Amount (mm) }\end{array}$ & $\begin{array}{c}\text { 比例 } \\
\text { Proportion (\%) }\end{array}$ & $\begin{array}{c}\text { 灌溉量 } \\
\text { Amount (mm) }\end{array}$ & $\begin{array}{c}\text { 比例 } \\
\text { Proportion (\%) }\end{array}$ & $\begin{array}{c}\text { 降水量 } \\
\text { Amount (mm) }\end{array}$ & $\begin{array}{c}\text { 比例 } \\
\text { Proportion }(\%)\end{array}$ \\
\hline \multicolumn{8}{|c|}{ 山农 15 Shannong 15} \\
\hline LSM & $372.8 \mathrm{e}$ & $230.0 \mathrm{a}$ & $61.7 \mathrm{a}$ & - & - & 142.8 & $38.3 \mathrm{a}$ \\
\hline MSM & $437.5 \mathrm{~b}$ & $228.1 \mathrm{a}$ & $52.1 \mathrm{~b}$ & $66.6 \mathrm{c}$ & $15.2 \mathrm{c}$ & 142.8 & $32.6 \mathrm{~b}$ \\
\hline HSM & $450.4 \mathrm{a}$ & $201.0 \mathrm{c}$ & $44.6 \mathrm{~d}$ & $106.6 \mathrm{a}$ & $23.7 \mathrm{a}$ & 142.8 & $31.7 \mathrm{~b}$ \\
\hline \multicolumn{8}{|c|}{ 济麦 22 Jimai 22} \\
\hline LSM & $369.4 \mathrm{e}$ & $226.6 \mathrm{a}$ & $61.3 \mathrm{a}$ & - & - & 142.8 & $38.7 \mathrm{a}$ \\
\hline MSM & $430.6 \mathrm{c}$ & $217.5 \mathrm{~b}$ & $50.5 \mathrm{c}$ & $70.3 \mathrm{c}$ & $16.3 \mathrm{c}$ & 142.8 & $33.2 \mathrm{~b}$ \\
\hline HSM & $454.1 \mathrm{a}$ & $209.3 \mathrm{bc}$ & $46.1 \mathrm{~d}$ & $102.0 \mathrm{a}$ & $22.5 \mathrm{a}$ & 142.8 & $31.5 \mathrm{~b}$ \\
\hline \multicolumn{8}{|c|}{ 烟农 21 Yannong 21} \\
\hline LSM & $371.5 \mathrm{e}$ & $228.7 \mathrm{a}$ & $61.6 \mathrm{a}$ & - & - & 142.8 & $38.4 \mathrm{a}$ \\
\hline MSM & $422.2 \mathrm{~d}$ & $212.4 \mathrm{~b}$ & $50.3 \mathrm{c}$ & $67.0 \mathrm{c}$ & $15.9 \mathrm{c}$ & 142.8 & $33.8 \mathrm{~b}$ \\
\hline HSM & $441.5 \mathrm{ab}$ & $204.6 \mathrm{c}$ & $46.3 \mathrm{~d}$ & $94.1 \mathrm{~b}$ & $21.3 \mathrm{~b}$ & 142.8 & $32.4 \mathrm{~b}$ \\
\hline
\end{tabular}

LSM: 低土壤含水量; MSM: 中土壤含水量; HSM: 高土壤含水量。同一列数据后不同字母表示差异达 0.05 显著水平。

LSM: low soil moisture; MSM: medium soil moisture; HSM: high soil moisture. Values followed by different letters in the same column are significantly different at $P<0.05$.

表 5 不同品种小麦各生育阶段的耗水量、耗水模系数和日耗水量(2008-2009)

Table 5 Water consumption amount, water consumption percentage and water consumption per day of different wheat cultivates in different growing periods in 2008-2009

\begin{tabular}{|c|c|c|c|c|c|c|c|c|c|}
\hline \multirow{2}{*}{$\begin{array}{c}\text { 处理 } \\
\text { Treatment }\end{array}$} & \multicolumn{3}{|c|}{ 播种至拔节期 Sowing-jointing } & \multicolumn{3}{|c|}{ 拔节至开花期 Jointing-anthesis } & \multicolumn{3}{|c|}{ 开花至成熟期 Anthesis-maturity } \\
\hline & $\mathrm{CA}(\mathrm{mm})$ & CP (\%) & $\mathrm{CD}(\mathrm{mm})$ & $\mathrm{CA}(\mathrm{mm})$ & CP (\%) & $\mathrm{CD}(\mathrm{mm})$ & $\mathrm{CA}(\mathrm{mm})$ & CP (\%) & $\mathrm{CD}(\mathrm{mm})$ \\
\hline
\end{tabular}

山农 15 Shannong 15

\begin{tabular}{|c|c|c|c|c|c|c|c|c|c|}
\hline LSM & $127.6 \mathrm{~b}$ & $34.2 \mathrm{~b}$ & $0.7 \mathrm{~b}$ & $110.0 \mathrm{c}$ & $29.5 \mathrm{~b}$ & $3.6 \mathrm{c}$ & $135.2 \mathrm{c}$ & $36.3 \mathrm{~b}$ & $3.5 \mathrm{c}$ \\
\hline MSM & $127.6 \mathrm{~b}$ & $29.2 \mathrm{~d}$ & $0.7 \mathrm{~b}$ & $144.7 \mathrm{a}$ & $33.1 \mathrm{a}$ & $4.7 \mathrm{a}$ & $165.2 \mathrm{~b}$ & $37.8 \mathrm{~b}$ & $4.2 \mathrm{~b}$ \\
\hline HSM & $127.6 \mathrm{~b}$ & $28.3 \mathrm{e}$ & $0.7 \mathrm{~b}$ & $144.7 \mathrm{a}$ & $32.1 \mathrm{a}$ & $4.7 \mathrm{a}$ & $178.1 \mathrm{a}$ & $39.5 \mathrm{a}$ & $4.6 \mathrm{a}$ \\
\hline
\end{tabular}

济麦 22 Jimai 22

\begin{tabular}{|c|c|c|c|c|c|c|c|c|c|}
\hline LSM & $143.9 \mathrm{a}$ & $39.0 \mathrm{a}$ & $0.8 \mathrm{a}$ & $87.0 \mathrm{~d}$ & $23.6 \mathrm{~d}$ & $2.8 \mathrm{e}$ & $138.5 \mathrm{c}$ & $37.5 \mathrm{~b}$ & $3.6 \mathrm{c}$ \\
\hline MSM & $143.9 \mathrm{a}$ & $33.4 \mathrm{~b}$ & $0.8 \mathrm{a}$ & $125.8 \mathrm{~b}$ & $29.2 \mathrm{~b}$ & $4.1 \mathrm{~b}$ & $161.0 \mathrm{~b}$ & $37.4 \mathrm{~b}$ & $4.1 \mathrm{~b}$ \\
\hline HSM & $143.9 \mathrm{a}$ & $31.7 \mathrm{c}$ & $0.8 \mathrm{a}$ & $125.8 \mathrm{~b}$ & $27.7 \mathrm{c}$ & $4.1 \mathrm{~b}$ & $184.4 \mathrm{a}$ & $40.6 \mathrm{a}$ & $4.7 \mathrm{a}$ \\
\hline
\end{tabular}

烟农 21 Yannong 21

\begin{tabular}{|c|c|c|c|c|c|c|c|c|c|}
\hline LSM & $139.9 \mathrm{a}$ & $37.7 \mathrm{a}$ & $0.8 \mathrm{a}$ & $93.9 \mathrm{~d}$ & $25.3 \mathrm{c}$ & $3.0 \mathrm{~d}$ & $137.7 \mathrm{c}$ & $37.1 \mathrm{~b}$ & $3.5 \mathrm{c}$ \\
\hline MSM & 139.9 a & $33.2 \mathrm{~b}$ & $0.8 \mathrm{a}$ & $125.0 \mathrm{~b}$ & $29.6 \mathrm{~b}$ & $4.0 \mathrm{~b}$ & $157.3 \mathrm{~b}$ & $37.3 \mathrm{~b}$ & $4.0 \mathrm{~b}$ \\
\hline HSM & $139.9 \mathrm{a}$ & $31.7 \mathrm{c}$ & $0.8 \mathrm{a}$ & $125.0 \mathrm{~b}$ & $28.3 \mathrm{~b}$ & $4.0 \mathrm{~b}$ & $176.6 \mathrm{a}$ & $40.0 \mathrm{a}$ & $4.5 \mathrm{a}$ \\
\hline
\end{tabular}

LSM: 低土壤含水量; MSM: 中土壤含水量; HSM: 高土壤含水量; CA: 各生育阶段麦田耗水量; CP: 耗水模系数; CD: 日耗水

量。同一列数据后不同字母表示差异达 0.05 显著水平。

LSM: low soil moisture; MSM: medium soil moisture; HSM: high soil moisture; CA: water consumption amount in growth stages; CP: water consumption percentage; $\mathrm{CD}$ : water consumption amount per day. Values in the same column followed by different letters are significantly different at $P<0.05$.

较高的品种山农 15 , 开花期干物质积累量亦较高, 此类品种可通过提高开花前贬藏同化物向籽粒的转 运量和经济系数获得高产, 达到节水高产的目的。
本试验条件下, 综合考虑䊏粒的产量、水分利用率 及灌水量, I组的品种为超高产节水品种, MSM处理 是节水高产的最优处理。 
表 6 不同品种小麦花后营养器官干物质积累量和干物质再分配量(2008-2009)

Table 6 Dry matter accumulation and translocation amount of vegetative organ after anthesis in different wheat cultivars in 2008-2009

\begin{tabular}{|c|c|c|c|c|c|c|c|c|}
\hline \multirow{2}{*}{$\begin{array}{c}\text { 处理 } \\
\text { Treatment }\end{array}$} & \multirow{2}{*}{$\begin{array}{l}\text { DMAAA } \\
\left(\mathrm{kg} \mathrm{hm}^{-2}\right)\end{array}$} & \multirow{2}{*}{$\begin{array}{l}\text { DMAAM } \\
\left(\mathrm{kg} \mathrm{hm}^{-2}\right)\end{array}$} & \multirow{2}{*}{$\begin{array}{l}\mathrm{HI} \\
(\%)\end{array}$} & \multicolumn{3}{|c|}{ DMDBA } & \multicolumn{2}{|c|}{ DMAAA } \\
\hline & & & & $\mathrm{TA}\left(\mathrm{kg} \mathrm{hm}^{-2}\right)$ & TR (\%) & CG (\%) & $\mathrm{AA}\left(\mathrm{kg} \mathrm{hm}^{-2}\right)$ & CG (\%) \\
\hline \multicolumn{9}{|c|}{ 山农 15 Shannong 15} \\
\hline LSM & $11024.9 \mathrm{c}$ & $15925.5 \mathrm{c}$ & $50.8 \mathrm{a}$ & $3182.2 \mathrm{a}$ & $28.9 \mathrm{a}$ & $39.4 \mathrm{a}$ & $4900.7 \mathrm{e}$ & $60.6 \mathrm{f}$ \\
\hline MSM & $13217.4 \mathrm{a}$ & $19348.1 \mathrm{a}$ & $47.4 \mathrm{c}$ & $3032.7 \mathrm{~b}$ & $22.9 \mathrm{c}$ & $33.1 \mathrm{~b}$ & $6130.7 \mathrm{c}$ & $66.9 \mathrm{e}$ \\
\hline HSM & $13132.8 \mathrm{a}$ & $19898.0 \mathrm{a}$ & $46.1 \mathrm{~d}$ & $2416.9 \mathrm{~d}$ & $18.4 \mathrm{~d}$ & $26.3 \mathrm{~d}$ & $6765.2 \mathrm{~b}$ & $73.7 \mathrm{c}$ \\
\hline \multicolumn{9}{|l|}{ 济麦 22 Jimai 22} \\
\hline LSM & $11031.9 \mathrm{c}$ & $15873.7 \mathrm{c}$ & $47.5 \mathrm{c}$ & $2696.8 \mathrm{c}$ & $24.5 \mathrm{~b}$ & $35.8 \mathrm{~b}$ & $4841.8 \mathrm{e}$ & $64.2 \mathrm{e}$ \\
\hline MSM & $12509.3 \mathrm{~b}$ & $19048.2 \mathrm{a}$ & $45.5 \mathrm{e}$ & $2121.4 \mathrm{f}$ & $17.0 \mathrm{e}$ & $24.5 \mathrm{e}$ & $6538.9 \mathrm{~b}$ & $75.5 \mathrm{~b}$ \\
\hline HSM & $12548.3 \mathrm{~b}$ & 19897.7 a & $45.3 \mathrm{e}$ & $1656.4 \mathrm{f}$ & $13.2 \mathrm{f}$ & $18.4 \mathrm{f}$ & $7349.4 \mathrm{a}$ & $81.6 \mathrm{a}$ \\
\hline \multicolumn{9}{|c|}{ 烟农 21 Yannong 21} \\
\hline LSM & $9906.0 \mathrm{~d}$ & $14970.4 \mathrm{e}$ & $48.9 \mathrm{~b}$ & $2262.1 \mathrm{e}$ & $22.8 \mathrm{c}$ & $30.9 \mathrm{c}$ & $5064.4 \mathrm{~d}$ & $69.1 \mathrm{~d}$ \\
\hline MSM & $12172.7 \mathrm{~b}$ & $18151.9 \mathrm{~b}$ & $44.9 \mathrm{f}$ & $2171.3 \mathrm{f}$ & $17.8 \mathrm{~d}$ & $26.6 \mathrm{~d}$ & $5979.2 \mathrm{c}$ & $73.4 \mathrm{c}$ \\
\hline HSM & $12217.3 \mathrm{~b}$ & $18903.6 \mathrm{~b}$ & $44.0 \mathrm{~g}$ & $1631.5 \mathrm{~g}$ & $13.4 \mathrm{f}$ & $19.6 \mathrm{f}$ & $6686.2 \mathrm{~b}$ & $80.4 \mathrm{a}$ \\
\hline
\end{tabular}

DMAAA: 开花期干物质积累量; DMAAM: 成熟期干物质积累量; HI: 经济系数; DMDBA: 营养器官花前咜藏干物质; TA: 转运 量; TR: 转运率; CG: 籽粒贡献率; DMAAA: 花后同化的干物质; AA: 积累量; LSM: 低土壤含水量; MSM: 中土壤含水量; HSM: 高 土壤含水量。同一列数据后不同字母表示差异达 0.05 显著水平。

DMAAA: dry matter accumulative amount at anthesis stage; DMAAM: dry matter accumulative amount at maturity stage; HI: harvest index; DMDBA: dry matter deposit before anthesis; TA: translocation amount; TR: translocation ratio; CG: contribution to grain; DMAAA: dry matter assimilated after anthesis; AA: assimilation amount; LSM: low soil moisture; MSM: medium soil moisture; HSM: high soil moisture. Values in the same column followed by different letters are significantly different at $P<0.05$.

\section{3 讨论}

已有很多试验探讨小麦节水高产的灌水量和灌 水次数 ${ }^{[12,22-25]}$ 。研究表明, 在一定范围内增加灌水量 具有增产效应, 但过多灌水导致籽粒产量和水分利 用率显著降低 ${ }^{[24-25]}$ 。表明在一定灌水量范围内, 产 量和水分利用率有一个最佳值, 且不同品种获得最 佳值时的灌水量和灌水次数不同 ${ }^{[12]}$ 。樊廷录等 ${ }^{[26]}$ 用 12 个旱地品种研究, 发现不同品种产量和水分利用 率存在显著差异, 冯广龙和刘昌明 ${ }^{[27]}$ 指出根系的分 布与发育显著影响小麦品种的水分利用率。水分利 用率与产量呈正相关, 种植具有较高产量潜力的品 种是提高水分利用率并达到节水效果的一种有效途 径 ${ }^{[28]}$ 。在华北地区, 正常年(全生育期降水量 $140 \mathrm{~mm}$ ) 小麦全生育期灌溉60 mm、干旱年(全生育期降水量 $73.8 \mathrm{~mm}$ ) 灌溉 $150 \mathrm{~mm}$ 、丰水年 $($ 全生育期降水量 $200.8 \mathrm{~mm}$ ) 不灌水可获得较高的产量和水分利用率 ${ }^{[29]}$ 。 本试验以 14 个水浇地小麦品种为试验材料, 在小麦 全生育期降水量 $169.8 \mathrm{~mm}$ 条件下, 利用测墑补灌的 方法, 将其聚类分为 3 组, 其中超高产高水分利用率 组(I组)山农15、临麦4号等在MSM条件下获得较高 产量和水分利用率, 超高产中水分利用率组(II组)济
麦22、汶农6号等在HSM条件下获得较高产量, 高产 低水分利用率组(III组)烟农 21、良星99等则在MSM 条件下产量和水分利用率较高, 但显著低于I组和II 组。本研究明确了I组在MSM条件下获得较高的产量 和水分利用率, 为选用节水高产小麦品种及其获得 节水高产的适宜土壤相对含水量的调控提供了依 据。不同年际间小麦各生育时期降水量不同对小麦 产量和水分利用效率也产生显著影响, 有待进一步 研究。

在高产条件下，冬小麦的产量和耗水量之间呈 非线性关系 ${ }^{[30-31],} \mathrm{Li}$ 等 ${ }^{[32]}$ 认为在中等干旱条件下冬 小麦耗水量大幅度下降, 而产量下降幅度较小, 有 利于水分利用率的提高。不同小麦品种比较, 在灌 水条件相同时品种间全生育期耗水量无显著差异, 产量和水分利用率差异显著 ${ }^{[33]}$; 另有研究指出, 即 使全生育期的总耗水量相同, 各生长阶段的分配比 例不同, 产量亦不相同 ${ }^{[34]}$, 节水抗旱型小麦耗水动 态与喜肥型品种相似，表现前期耗水少，中后期耗 水多的特点 ${ }^{[35]}$ 。本试验结果表明, 不同品种间的总 耗水量及阶段耗水量均存在差异, 在相同灌水条件 下，山农 15 和济麦 22 的总耗水量显著高于烟农 21 ; 山农15拔节前耗水少, 拔节至开花期耗水量较高, 
开花至成熟期, 与济麦22和烟农 21 无显著差异, 表 明山农 15 前期耗水少, 中后期耗水多, 有利于穗器 官的发育和䊏粒的充分灌浆, 是其获得节水高产的 主要原因。山农15拔节至开花期耗水量较高主要是 因为其利用了较多的土壤咜水, 这是否与此类品种 的生长特性和根系分布有关，有待进一步研究。

小麦在某些生育时期水分相对不足或有限亏缺, 有利于同化物向籽粒转运, 提高经济系数 ${ }^{[36]}$, 灌浆 期水分亏缺可促进营养器官开花前䛎藏干物质向籽

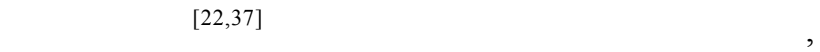
产量和水分利用率的提高主要是由于增加了灌浆期 叶片的净光合速率和光合功能持续期, 促进花前储 存碳库的再转运, 显著提高了经济系数 ${ }^{[38]}$ 。例如, 与 西风20相比, 抗旱品种石家庄 8 号能够在较强干旱 胁迫下生产较高的干物质获得高产 ${ }^{[29]}$ 。本研究结果 为, MSM和HSM条件下, 山农 15 的开花期干物质积 累量显著高于济麦22和烟农 21 , 成熟期则表现为山 农 15 和济麦 22 显著高于烟农 21 , 经济系数为山农 $15>$ 济麦 $22>$ 烟农 21 。表明山农 15 在开花期土壤相对 含水量为 $65 \% \sim 70 \%$ 条件下, 开花期和成熟期较高的 干物质积累量、较高经济系数和较高的营养器官开 花前陉藏同化物向籽粒的转运量和转运率及对籽粒 的贡献率，是其获得节水高产的主要原因。

\section{4 结论}

以小麦籽粒产量和水分利用率为指标, 将 14 个 小麦品种分为超高产高水分利用率组 (I 组)、超高产 中水分利用率组(II 组)和高产低水分利用率组(III 组)。在 MSM 条件下, I 组品种山农 15 的土壤咜水消 耗量及占总耗水量的比例、拔节至开花期的耗水 量、开花期干物质积累量、经济系数以及开花前咜 藏同化物向籽粒的转运量均显著高于 II 组品种济 麦22和 III 组品种烟农 21 。在本试验条件下, I 组品 种为超高产节水品种, MSM 处理是节水高产的最 优处理。

\section{References}

[1] Liu B-C(刘布春), Mei X-R(梅旭荣), Li Y-Z(李玉中), Yang Y-L(杨有禄). The connotation and extension of agricultural water resources security. Sci Agric Sin (中国农业科学), 2006, 39(5): 947-951 (in Chinese with English abstract)

[2] Kang S-Z(康绍忠), Hu X-T(胡笑涛), Cai H-J(蔡焕杰), Feng S-Y(冯绍元). New ideas and development tendency of theory for water saving in modern agriculture and ecology. J Hydraulic Eng (水利学报), 2004, (12): 1-7 (in Chinese with English abstract)

[3] Sun H Y, Liu C M, Zhang X Y, Shen Y J, Zhang Y Q. Effects of irrigation on water balance, yield and WUE of winter wheat in the North China Plain. Agric Water Manag, 2006, 85: 211-218

[4] Rajala A, Hakala K, Mäkelä P, Muurinen S, Peltonen-Sainio P. Spring wheat response to timing of water deficit through sink and grain filling capacity. Field Crops Res, 2009, 114: 263-271

[5] Panda R K, Behera S K, Kashyap P S. Effective management of irrigation water for wheat under stressed conditions. Agric Water Manag, 2003, 63: 37-56

[6] Men H-W(门洪文), Zhang Q(张秋), Dai X-L(代兴龙), Cao Q(曹 倩), Wang C-Y(王成雨), Zhou X-H(周晓虎), He M-R(贺明荣). Effects of different irrigation modes on winter wheat grain yield and water-and nitrogen use efficiency. Chin J Appl Ecol (应用生 态学报), 2011, 22(10): 2517-2523 (in Chinese with English abstract)

[7] Zhi H-M(支虎明), Liu J-H(刘建华). Influence of limited supplemental irrigation at different periods (stages) on wheat yield, water use efficiency and economic efficiency. Chin Agric Sci Bull (中国农学通报), 2011, 27(9): 314-319 (in Chinese with English abstract)

[8] Virgona J W, Barlow E W R. Drought stress induces changes in the nonstructural carbohydrate composition of wheat system. Aust J Physiol, 1989, 18: 239-247

[9] Zhang Y-Q(张雅倩), Lin Q(林琪), Jiang W(姜雯), Liu Y-G(刘义 国), Li L-Y(李玲燕). Drought-resistance characteristics of different fertilizer and water types of wheat under water stress. Acta Agric Boreali-Sin (华北农学报), 2010, 25(6): 205-210 (in Chinese with English abstract)

[10] Rizza F, Badeck F W, Cattivelli L, Lidestri O, Fonzo N D, Stanca A M. Use of a water stress index to identify barley genotypes adapted to rained and irrigated conditions. Crop Sci, 2004, 44: 2127-2137

[11] Shao H B, Liang Z S, Shao M G, Sun S M, Hu Z M. Investigation on dynamic changes of photosynthetic characteristics of 10 wheat (Triticum aestivum L.) genotypes during two vegetative-growth stages at water deficit. Colloids Surfaces B: Biointerfaces, 2005, 43: $221-227$

[12] Dong B-D(董宝娣), Zhang Z-B(张正斌), Liu M-Y(刘孟雨), Zhang Y-Z(张依章), Li Q-Q(李全起), Shi L(石否), Zhou Y-T(周 永田). Water use characteristics of different wheat varieties and their responses to different irrigation schedulings. Trans CSAE (农业工程学报), 2007, 23(9): 27-33 (in Chinese with English abstract)

[13] Liu W-D(刘万代), Yin J(尹钧), Zhu G-J(朱高纪). Effects of leaf removal on dry matter accumulation and grain yield in different spike-type wheat varieties. Sci Agric Sin (中国农业科学), 2007, 40(7): 1353-1360 (in Chinese with English abstract)

[14] Dai Z-M(戴忠民)，Wang Z-L(王振林)，Gao F-J(高凤菊)， Li W-Y(李文阳), Yan S-H(闯素辉), Cai R-G(蔡瑞国), Zhang M (张敏), Yin Y-P(尹燕枰). Characterization of starch accumulation and activities of enzymes involved in starch synthesis in grains of wheat cultivars differing in spike types field-grown in irrigation and rainfed conditions. Acta Agron Sin (作物学报), 2007, 33(4): 682-685 (in Chinese with English abstract)

[15] Dong B-D(董宝娣), Shi C-H(师长海), Qiao Y-Z(乔匀周), Yang J(杨静), Zhai H-M(翟红梅), Li D-X(李东晓), Liu M-Y(刘孟雨). Analysis of water use efficiency of different winter wheat culti- 
vars under different irrigation schemes on the basis of yield. Chin $J$ Eco-Agric (中国生态农业学报), 2011，19(5)：1096-1103 (in Chinese with English abstract)

[16] Luo H-Y(骆洪义), Ding F-J(丁方军). Laboratory Guide for Pedology (土壤学实验). Chengdu: Chengdu Science and Technology University Press, 1995. p 91 (in Chinese)

[17] Shan L(山仑), Kang S-Z(康绍忠), Wu P-T(吴普特). Water-Saving Agriculture in China (中国节水农业). Beijing: China Agriculture Press, 2004. pp 229-230 (in Chinese)

[18］ Tao Y-F(陶毓汾), Wang L-X(王立祥), Han S-F(韩仕峰). Water Production Potential and Development of Dryland Field in Northern China (中国北方旱农地区水分生产潜力与开发). Beijing: China Meteorological Press, 1993. pp 63-157(in Chinese)

[19] Despo K P, Gagianas A A. Nitrogen and dry matter accumulation, remobilization, and losses for Mediterranean wheat during grain filling. Agron J, 1991, 83: 864-870

[20] Hussain G, Al-Jaloud A A. Effect of irrigation and nitrogen on water use efficiency of wheat in Saudi Arabia. Agric Water Manag, 1995, 27: 143-53

[21] Han Y-L(韩燕来), Jie X-D(介晓否), Tan J-F(谭金芳), Guo T-C(郭天财), Zhu Y-J(朱云集), Wang C-Y(王晨阳), Xia G-J(夏 国军), Liu Z(刘征). Studies on absorption, distribution and translocation of N, P and $\mathrm{K}$ of super-high yield winter wheat. Acta Agron Sin (作物学报), 1998, 24(6): 908-915 (in Chinese with English abstract)

[22] Yang J C, Zhang J H, Huang Z L, Zhu Q S, Wang L. Remobilization of carbon reserves is improved by controlled soil-drying during grain filling of wheat. Crop Sci, 2000, 40: 1645-1655

[23] Panda R K, Behera S K, Kashyap P S. Effective management of irrigation water for wheat under stressed conditions. Agric Water Manag, 2003, 63: 37-56

[24] Zhang X Y, Chen S Y, Sun H Y. Dry matter, harvest index, grain yield and water use efficiency as affected by water supply in winter wheat. Irrig Sci, 2008, 7: 1-10

[25] Sun H Y, Shen Y J, Yu Q, Gerald F, Zhang Y Q, Liu C M, Zhang $X$ Y. Effect of precipitation change on water balance and WUE of the winter wheat-summer maize rotation in the North China Plain. Agric Water Manag, 2010, 97: 1139-1145

[26] Fan T-L(樊廷录), Ma M-S(马明生), Wang S-Y(王淑英), Li S-Z(李尚中), Zhao G(赵刚). A study on the relationship between stem soluble sugar with grain yield and water use efficiency in different winter wheat under limited irrigation condition. Sci Agric Sin (中国农业科学), 2010, 43(12): 2428-2434 (in Chinese with English abstract)

[27] Feng G-L(冯广龙), Liu C-M(刘昌明). Analys is of root system growth in relation to soil water extraction pattern by winter wheat under water limiting conditions. $J$ Nat Resour (自然资源学报), 1998, 13(3): 234-240 (in Chinese with English abstract)
[28] Zhang X Y, Chen S Y, Sun H Y, Wang Y M, Shao L W. Water use efficiency and associated traits in winter wheat cultivars in the North China Plain. Agric Water Manag, 2010, 97: 1117-1125

[29] Dong B D, Shi L, Shi C H, Qiao Y Z, Liu M Y, Zhang Z B. Grain yield and water use efficiency of two types of winter wheat cultivars under different water regimes. Agric Water Manag, 2011, 99: 103-110

[30] Ning D-F(宁东峰), Li Z-J(李志杰), Sun W-Y(孙文彦), Ma W-P(马卫萍), Huang S-W(黄绍文), Zhao B-Q(赵秉强). Effects of water-saving irrigation on water consumption and photosynthetic characteristics of winter wheat in Huang-Huai-Hai area of China. Plant Nutr Fert Sci (植物营养与肥料学报), 2010, 16(4): 852-858 (in Chinese with English abstract)

[31] Zhang S-Q(张胜全), Fang B-T(方保停), Wang Z-M(王志敏), Zhou S-L(周顺利), Zhang Y-H(张英华). Influence of different spring irrigation treatments on water use and yield formation of late-sowing winter wheat. Acta Ecol Sin (生态学报), 2009, 29(4): 2035-2044 (in Chinese with English abstract)

[32] Li F M, Liu X L, Guo A H. Effects of early soil moisture distribution on the dry matter partition between root and shoot of winter wheat. Agric Water Manag, 2001, 49: 163-171

[33] Wang K-W(王克武), Wang Z-P(王志平), Zheng Y-L(郑雅莲), Zhang N(张娜), Zhu Q-Y(朱青艳). Selection of wheat varieties with high WUE and study on laws of their water consumption. Agric Res Arid Areas (干旱地区农业研究), 2009, 27(2): 69-73 (in Chinese with English abstract)

[34] Zhou L-Y(周凌云). Water supply and potential productivity in rainfed wheat field in Fengqiu region. Acta Pedol Sin (土壤学报), 1993, 30(3): 297-303 (in Chinese with English abstract)

[35] Sui P(隋鹏), Zhang H-L(张海林), Xu C(许翠), Gao W-S(高旺 盛). Comparison of soil water consumption characteristics between drought-resistant and water-liking wheat varieties. Agric Res Arid Areas (干旱地区农业研究), 2005, 23(4): 26-31, 57 (in Chinese with English abstract)

[36] Kang S Z, Zhang L, Liang Y L, Hu Y T, Cai H J, Gu B J. Effects of limited irrigation on yield and water use efficiency of winter wheat in the Loess Plateau of China. Agric Water Manag, 2002, 55: $203-216$

[37] Ercoli L, Lulli L, Mariotti M, Masoni A, Arduini I. Post-anthesis dry matter and nitrogen dynamics in durum wheat as affected by nitrogen supply and soil water availability. Eur J Agron, 2008, 28: $138-147$

[38] Hu M-Y(胡梦芸), Zhang Z-B(张正斌), Xu P(徐萍), Dong B-D(董宝娣), Li W-Q(李魏强), Li J-J(李景娟). Relationship of water use efficiency with photoassimilate accumulation and transport in wheat under deficit irrigation. Acta Agron Sin (作物 学报), 2007, 33 (11): 1884-1891 (in Chinese with English abstract) 